\title{
Suplementation of Antibiotic Growth Promoters versus Supplementation of Functional Oils in Broiler Breeders: Performance and Offspring Effects
}

\section{Euthor(s)}

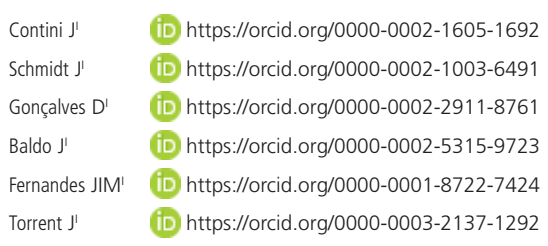

Laboratório de Experimentação Avícola UFPR Department of Animal Science, Rua Pioneiro, 2153, Palotina, PR, Brazil, 85950-000.

Oligo Basics USA LLC 840 Katahdin Way, Cary, North Carolina 27519, United States.

\section{-Mail Address}

Corresponding author e-mail address Joan Torrent

Oligo Basics USA LLC 840 Katahdin Way, Cary، North Carolina 27519, United States.

Phone: +1 952 451-6968

Email: jtorrent@oligobasics.com

\section{-Keywords}

Broiler breeders, functional oils, growth promoters, offspring effects.

\section{ABSTRACT}

The effects of either functional oil or antibiotic growth promoter supplementation on broiler breeders and their offspring were assessed in 2 experiments. In Experiment I, 16,400 broiler breeders (22 weeks old) were distributed into 2 experimental groups, one supplemented with 1,500 ppm of a commercial blend of functional oils (active ingredients: cashew nutshell liquid and castor oil) and the other with 500 ppm of bacitracin methylene disalicylate (BMD). Laying rates, fertility, embryonic mortality, and egg characteristics were measured from weeks 25 to 46. In Experiment II, 720 one day old male chicks, born from 42 week old breeders, from each one of the two treatments in Experiment I, were supplemented with either $10 \mathrm{ppm}$ of enramycin or 1,500 ppm of the same functional oils as in Experiment I to study whether there were any maternal diet carry-over effects. Functional oils decreased embryonic mortality from $11.01 \%$ to $9.64 \%(P<0.03)$ when compared to BMD. However, a functional oil link increase in egg weight did not result in either heavier chicks or statistically significant better offspring performance. Nonetheless, a possible benefit of the functional oil supplementation on the offspring weight at $42 \mathrm{~d}$ cannot be excluded. The type of additive supplemented during Experiment II did not affect any performance parameters. In conclusion, functional oils could successfully replace antibiotic growth promoters in broiler breeders as embryonic mortality decreased. Finally, when functional oils were supplemented to the offspring, the performance was similar to that of a commonly used antibiotic growth promoter.

\section{INTRODUCTION}

The use of antibiotic growth promoters is a widespread strategy to increase broiler breeder performance. The expected improvements are supposed to be derived from a better utilization of the limited feed allowance (Bozkurt et al., 2009). However, antibiotic growth promoters have been banned in the European Union since 2006, and most countries are implementing either total or partial bans on their use. Therefore, viable alternatives are needed to maintain productivity.

Nutritional, pathological, and environmental factors have been shown to increase free radical production in broiler breeders (Birendra and Jha, 2019; Hu et al., 2019). Free radicals negatively affect fertility and hatchability (Breque et al., 2003; Pappas, 2005) and the supplementation of antioxidants has been shown to counteract these negative effects (Rosa et al., 2012) and improve embryonic development (Surai et al., 2019). Whereas some functional oils such as cashew nut shell liquid have been shown to have antioxidant activity (Trevisan et al., 2005), others, like castor oil have been shown to be anti-inflammatory (Vieira et al., 2001) and have activity against some fungi and gram positive bacteria (Novak et al., 1961). It is probably because all of these 
activities that a commercial mixture of cashew nut shell liquid and castor oil has been shown to reduce inflammation in coccidiosis challenged birds (Moraes et al., 2019) and the negative effects of heat stress (Torrent et al., 2019). Also, functional oils have been shown to increase energy utilization in broiler diets (Bess et al., 2012; Murakami et al., 2014). Because of all these effects, functional oils should favorably affect the breeder's reproductive parameters or, at least, yield performance results similar to those of antibiotic growth promoters.

Supplementation of functional oils may also affect offspring performance. For example, Koedijk et al., (2015) showed that chick weight was significantly heavier at hatch and that average daily gain improved significantly in young breeder offspring when supplemented with polyphenols, which are known antioxidants. Therefore, the objective of this paper is: 1) to compare the performance of broiler breeders supplemented with either an antibiotic growth promoter or a commercial blend of functional oils (active ingredients: cashew nut shell liquid and castor oil), 2) to evaluate whether these effects carried over to the offspring performance, 3) to evaluate how the supplementation with either functional oils or antibiotic growth promoters affected broiler performance, and 4) to study whether there was an interaction between the maternal and the offspring supplementation that affected performance.

\section{MATERIALS AND METHODS}

\section{Experiment I}

All the procedures used in this experiment were approved by the Universidade Federal do Paraná in Palotina Animal Care Committee and were in accordance with the guidelines outlined by the University Animal Care Committee.

\section{Animals}

A total of 16,400 broiler breeders and 1,722 roosters (22 week old), from a commercial poultry integrator, were randomly assigned to two different bird houses (8,200 breeders and 861 roosters each) in the same breeder complex. Historically, both houses had previously been shown to consistently yield non significant different results in breeder performance. The birds were distributed at 23 weeks of age into 2 experimental groups with similar body weight and uniformity and were reared following the guidelines of Aviagen for Ross AP 95 broiler breeders.

\section{Feed and experimental additives}

Birds were fed corn-soybean-based mash diets (Table 1). The supply of the feed was strictly controlled, in accordance with the recommendations of the breeder company. One group received 1,500 ppm of a commercial blend of two functional oils, cashew nut shell liquid and castor oil (active components in the commercial product: $20 \%$ cardanol and 5\% cardol from cashew nut shell liquid and $9 \%$ ricinoleic acid from castor oil; Essential, Oligo Basics Agroind. Ltda, Cascavel, Brazil) supplemented in the diet using expanded vermiculite as a carrier, and the second group received the diet supplemented with 500 ppm of bacitracin methylene disalicylate (BMD). Roosters in both groups received the same diet, which was also supplemented with 500 ppm of BMD. During the whole period, the same male:female ratio was maintained in both houses.

\section{Housing of birds, collection and incubation of eggs}

The birds were placed in houses using negative pressure ventilation, with wood shavings litter floors, equipped with automatic drinkers, tube feeders for the hens, and a trough-type feeder for the roosters. Water was supplied for ad libitum consumption, and a photoperiod of 16L:8D was used during the study.

The laying rate was calculated weekly as the number of eggs produced per breeder per week per group divided by 7 and was measured from week 26 till week 46 of age. A total of 5,040 hatching eggs per treatment were collected on weeks 26, 30, 34, 38, 42, and 46 .

The incubation was performed in a commercial hatchery, in single-stage incubators, following company standards. Trolleys were identified and the eggs were incubated for $48 \mathrm{~h}$ and at that point, fertility was evaluated using the automatic vaccination scanner machine. At 18 days of incubation, the in ovo vaccination was performed for infectious bursal disease and Marek's disease, and the machine automatically removed and counted infertile eggs. Eggs previously vaccinated and therefore considered fertile were transferred to the hatchers in identified trays, where they remained until the 21 st day of incubation. On the 21st day of incubation after hatching, the total number of viable chicks and total non-hatched eggs per tray were counted in each treatment. Fertility was calculated based on the number of infertile eggs (removed by the vaccinating machine) according to the total number of eggs incubated on each floor of 
Table 1 - Ingredients and nutritional composition of the breeder diets in Experiment I.

\begin{tabular}{|c|c|c|c|c|}
\hline \multirow[b]{2}{*}{ Ingredients, \% } & \multicolumn{2}{|c|}{ Wk $24-42$} & \multicolumn{2}{|r|}{ Wk $42-46$} \\
\hline & $\mathrm{BMD}^{1}$ & Functional Oils & BMD & Functional Oils \\
\hline Corn & 63.74 & 63.74 & 71.41 & 71.42 \\
\hline Soybean oil & 1.77 & 1.77 & - & - \\
\hline Soybean meal & 19.33 & 19.33 & 19.08 & 19.08 \\
\hline Wheat mids & 6.00 & 6.00 & - & - \\
\hline Dicalcium phosphate & 1.09 & 1.09 & 1.05 & 1.05 \\
\hline Salt & 0.25 & 0.25 & 0.25 & 0.25 \\
\hline DL-Methione & 0.10 & 0.10 & 0.10 & 0.10 \\
\hline Choline chloride & 0.12 & 0.12 & 0.10 & 0.10 \\
\hline Sodium bicarbonate & 0.12 & 0.12 & 0.12 & 0.12 \\
\hline Limestone & 4.00 & 4.00 & 3.83 & 3.83 \\
\hline Limestone, $5 \mathrm{~mm}$ & 2.05 & 2.05 & 2.77 & 2.77 \\
\hline BMD & 0.05 & - & 0.05 & - \\
\hline Functional oils & - & 0.15 & - & 0.15 \\
\hline Vitamin-mineral premix ${ }^{2}$ & 0.50 & 0.50 & 0.50 & 0.50 \\
\hline \multicolumn{5}{|l|}{ Nutritional composition } \\
\hline Metabolizable Energy, kcal/kg & \multicolumn{2}{|c|}{2,850} & \multicolumn{2}{|r|}{2.850} \\
\hline Crude Protein, \% & \multicolumn{2}{|c|}{15.00} & \multicolumn{2}{|r|}{14.50} \\
\hline Ether Extract, \% & \multicolumn{2}{|c|}{4.46} & \multicolumn{2}{|r|}{2.81} \\
\hline Linoleic acid, \% & \multicolumn{2}{|c|}{2.20} & \multicolumn{2}{|r|}{1.29} \\
\hline $\mathrm{Ca}, \%$ & \multicolumn{2}{|c|}{2.80} & \multicolumn{2}{|r|}{3.00} \\
\hline Available P, \% & \multicolumn{2}{|c|}{0.40} & \multicolumn{2}{|r|}{0.38} \\
\hline Digestible Lys, \% & \multicolumn{2}{|c|}{0.658} & \multicolumn{2}{|r|}{0.637} \\
\hline AAS dig, $\%$ & \multicolumn{2}{|c|}{0.542} & \multicolumn{2}{|r|}{0.534} \\
\hline Digestible threonine, \% & \multicolumn{2}{|c|}{0.487} & \multicolumn{2}{|r|}{0.477} \\
\hline Digestible triptophne, \% & \multicolumn{2}{|c|}{0.153} & \multicolumn{2}{|r|}{0.144} \\
\hline Digestible leucine, \% & \multicolumn{2}{|c|}{1.218} & \multicolumn{2}{|r|}{1.229} \\
\hline Digestible isoleucine, \% & \multicolumn{2}{|c|}{0.566} & \multicolumn{2}{|r|}{0.555} \\
\hline Digestible arginine, \% & \multicolumn{2}{|c|}{0.879} & & 0.843 \\
\hline Choline, mg/kg & & & & 1479.02 \\
\hline $\mathrm{Na}, \%$ & & & & 0.170 \\
\hline $\mathrm{Cl}, \%$ & & & & 0.217 \\
\hline$K, \%$ & & & & 0.617 \\
\hline $\mathrm{Na}+\mathrm{K}+\mathrm{Cl}$ meq $/ 100 \mathrm{~g}$ & & & & 170.75 \\
\hline
\end{tabular}

'Bacitracin methylene disalicylate.

2Vitamin premix (Content per kg of premix): Vitamin:A 3,200 Ul; Vitamin D3: 900 Ul; Vitamin E: 32,000 mg; Vitamin K3: 1,000 mg;Vitamin B1: 1,000 mg; Vitamin B2: 2.400 mg; Vitamin B6: 1,600 mg; Vitamin B12: 8.000,40 g; Niacin: 13,000 mg; Pantothenic acid: 4.000 mg; Folic acid: 800 mg; Biotin: 79,992mcg; Antioxidant: 13,320. Mineral premix (Content per kg of premix): Cobalt: 100,10 mg; Iron: 12,000 mg; Copper: 4,000 mg; lodine: 400 mg; Zinc: 28,000 mg; Manganese: 26,000 mg; Selenium: 80mg; Phytase 100,000 FTU.

the egg trolley. Hatching was based on the number of chicks born according to the total number of incubated eggs. On weeks 25, 33, 41, and 49, 45 eggs per treatment were individually evaluated to determine the weight, specific gravity (determined by the flotation method using increments of .001), yolk weight, albumen weight, and shell weight and thickness. To measure the shell weight, the shells were washed and dried at room temperature for $72 \mathrm{~h}$ and were weighed using a precision digital scale $(0.001$ g). After weighing the shells, the shell thickness was measured at 3 points in the central region of each shell using a digital micrometer (Mitutoyo Sul Americana, São Paulo, Brazil). To evaluate the hatching rate of fertile eggs, fertility, and embryonic mortality, the non hatched eggs were submitted to embryo diagnostics. In this evaluation, the eggs were classified through a macroscopic visual examination as infertile; embryonic mortality at the first $72 \mathrm{~h}$ of incubation; embryonic mortality occurring between days 4 and 10 of incubation; embryonic mortality occurring between days 11 and 17 of incubation; embryonic mortality occurring between days 18 and 21 of incubation, including dead-pipped embryos; and pipped eggs that have broken the shell but not emerged at the time of removal of chicks from the hatcher and the embryo is still alive. Eggs with abnormal contents with green or black coloration and emitting rotten odors or eggs that exploded on opening were considered contaminated. 


\section{Experimental Design and Statistical Analysis}

Laying rates (\%) for both treatments were modeled according to the modified compartment logistic curvilinear model following the equation:

$y(t)=a e^{-b t} /\left[\left(1+e^{-c(t-d)}\right)\right]$ (Yang et al., 1989)

Where, $a=a$ scale parameter, $b=$ the rate of decrease in laying ability, $c=$ the reciprocal indicator of the variation in sexual maturity, and $d=$ the mean age of sexual maturity of the hens.

The comparison of the laying rates was done comparing the full logistic curvilinear model that used different coefficients for each treatment, to the reduced model that used the same coefficients for both treatments. This comparison is performed using the extra sum of squares that provide a means of formally testing whether one set of predictors (full model) is necessary given that another set is already in the model (reduced model). The method works by looking at the reduction in the residual sum of squares when the set of additional variables is added to the model. This change is divided by the number of degrees of freedom for the additional variables to produce a mean square. This mean square is compared to the residual mean square from the full model. The 2 models, one using just one set of logistic coefficients for both treatments and the other one using a different set of logistic coefficients for each treatment, were then compared using an F-statistic. (R packaged version 1.5.2).

It was not possible to directly calculate the relative number of chicks/breeder/day, as the different reproductive parameters were taken from samples and not from the total population of the birds studied. Therefore, a boostrapping technique was used to obtain the average percent of chicks/breeder/day during the period of the test and to compare the two treatments using Monte Carlo methods (Efron \& Tibshirani, 1993). The model was iterated 100,000 times, using a program written in Python, to obtain and compare the mean of the percentage number of chicks/breeder/day.

Laying rate, hatching rate, fertility, weekly mortality, embryonic mortality, contaminated and cracked eggs, and all the egg characteristics were the dependent variables. The independent variables were treatment and week of age. An analysis of variance with week as the repeated measure and treatment as the fixed factor was used to assess the reproductive and egg parameters differences ( $R$ packaged version 1.5.2). As percentage data are not normal they were arcsinetransformed before analysis to achieve normality.

\section{Experiment II}

This research was conducted at the Experimental Agronomic Station of the Federal University of the State of Paraná, in Palotina, Brazil, with the approval of the Institutional Animal Care and Use Committee, which oversees research with animals and poultry at university facilities.

\section{Birds and Housing}

A total of 720 one day old male chicks, born from 42 week old breeders, from each one of the two treatments in Experiment I, were assigned to either a treatment using $10 \mathrm{ppm}$ of enramycin or a treatment using 1,500 ppm of functional oils (Essential, Oligo Basics Agroind. Ltda, Cascavel, Brazil) as growth promoters. The birds were housed in a climate controlled house (exhaust fans, evaporative cooling pads, and brooder heaters), divided into 36 boxes of $3.52 \mathrm{~m}^{2}$, covered with reused litter. The thermal comfort temperature was maintained according to the age using a $300 \mathrm{~W}$ halogen lamp heating system. The birds received 24 hours of light from day 1 to day 21, and 16 hours of light and 8 of dark from day 21 till the end of the experiment. The experiment included a starter (1-21 days), a grower (22-35 days), and a finisher diet (36-42 days). The nutrient levels of the diets followed typical Brazilian industry standards (Table 2).

\section{Experimental Design and Statistical Analysis}

A $2 \times 2$ ANOVA with 2 breeder effects and 2 offspring effects (supplementation with either antibiotic growth promoters or with functional oils in both cases) was used to study treatment differences ( $R$ packaged version 1.5.2). Percentage data were arcsinetransformed before analysis to achieve normality.

\section{RESULTS AND DISCUSSION}

\section{Experiment I}

The comparison of the logistic curvilinear full model (including a different set of coefficients for each treatment) to the reduced model (including just one set of coefficients) showed that the full model was not superior to the reduced one and that, therefore, there were no differences for the laying rates between treatments. The equation obtained for the reduced model was (Figure 1):

$$
y(t)=125.94 e^{-0.012 t} /\left[\left(1+e^{-1.71(t-24.62)}\right)\right]
$$

Showing that birds reached sexual maturity at approximately 25 weeks of age and that the rate of decrease in laying ability was $1.2 \% /$ week, both values 
Contini J, Schmidt J, Gonçalves D, Baldo J, Fernandes JIM, Torrent J
Suplementation of Antibiotic Growth Promoters versus Supplementation of Functional Oils in Broiler Breeders: Performance and Offspring Effects
Table 2 - Ingredients and nutritional composition of the offspring diets of Experiment II.

\begin{tabular}{lccc}
\hline Ingredients, \% & Starter & Grower & Finisher \\
\hline Corn & 58.58 & 63.2 & 66.31 \\
\hline Soybean Oil & 1.30 & 1.20 & 1.80 \\
\hline Soybean Meal & 36.10 & 32.00 & 28.60 \\
\hline Dicalcium Phosphate & 1.16 & 1.08 & 0.94 \\
\hline Salt & 0.35 & 0.42 & 0.40 \\
\hline DL-Methionine & 0.29 & 0.24 & 0.22 \\
\hline Sodium Bicarbonate & 0.20 & - & - \\
\hline L-Threonine & 0.09 & 0.09 & 0.06 \\
\hline Kaolin' & 0.15 & 0.15 & 0.15 \\
\hline Limestone & 1.00 & 0.84 & 0.82 \\
\hline Vitamin and mineral premix & 0.30 & 0.30 & 0.30 \\
\hline Lysine - HCL & 0.37 & 0.36 & 0.32 \\
\hline Choline Chloride & 0.06 & 0.07 & 0.08 \\
\hline Anticoccidial & 0.05 & 0.05 & - \\
\hline Nutrient levels & & & \\
\hline Metabolizable energy, Kcal/kg & 2980 & 3050 & 3125 \\
\hline Crude Protein, \% & 21.93 & 20.36 & 18.96 \\
\hline Ether Extract, \% & 3.73 & 3.72 & 4.36 \\
\hline Crude Fiber, \% & 3.22 & 3.06 & 2.91 \\
\hline Ashes, \% & 6.12 & 5.44 & 5.06 \\
\hline Calcium, \% & 0.94 & 0.85 & 0.79 \\
\hline Available phosphorus, \% & 0.450 & 0.430 & 0.399 \\
\hline Digestible lysine, \% & 1.250 & 1.149 & 1.044 \\
\hline Digestible sulfur aminoacids, \% & 0.880 & 0.801 & 0.750 \\
\hline Digestible threonine, \% & 0.810 & 0.750 & 0.679 \\
\hline Digestible tryptophan, \% & 0.236 & 0.216 & 0.198 \\
\hline Digestible leucine, \% & 1.668 & 1.577 & 1.495 \\
\hline Digestible isoleucine, \% & 0.863 & 0.793 & 0.733 \\
\hline Digestible valine, \% & 0.923 & 0.856 & 0.797 \\
\hline Digestible arginine, \% & 1.329 & 1.217 & 1.122 \\
\hline Coline, mg/kg & 1598.21 & 1594.54 & 1549.48 \\
\hline Na, \% & 0.221 & 0.194 & 0.184 \\
\hline Cl, \% & 0.268 & 0.312 & 0.301 \\
\hline K, \% & 0.917 & 0.847 & 0.786 \\
\hline IRepced & & & \\
\hline
\end{tabular}

${ }^{1}$ Replaced entirely by Essential $\circledast$ (1.5kg/ton) or partially by Enramycin (125 g/ton) 2 Vitamin premix (per kg): Vit. A 4,000 IU; Vit. D3 1,167 IU; Vit. E 10,000 IU; Vit. K3 1000 mg; Vit. B1 1,000 mg; Vit. B2 2,666.67 mg; Vit. B6 1,667 mg;Vit. B12 6,666.67 mcg; Niacin 13,333.33 mg; Pantothenic Acid 6,000 mg; Folic Acid 833.33 mg; Biotin 80,000 mcg; Antioxidant 22,200 mg. Mineral premix (per kg): Iron 23,333.33 ppm; Copper 2,666.67 ppm; lodine 333.3 ppm; Zinc 33,333.33 ppm; Manganese 40,000 ppm; Selenium 80 ppm; Phytase 16,667 g/kg; Enzyme complex (xylanase, amylase and protease) $33.333 \mathrm{~g} / \mathrm{kg}$.

similar to what has previously been reported (de Avila et al., 2003, Lewis and Gous, 2003, respectively). The average laying rate was $77.4 \pm 13 \%$ across treatments for the whole length of the experiment. The hatching rate was analyzed the same way as laying rate and, again, there were no differences between the treatments, with an average rate of $73.2 \pm 16 \%$.

Fertility was not affected by the type of additive $93.73 \pm 0.82 \%$, however, there was a significant time-effect due to week $(p<0.01)$, and a possible but not significant type of additive by week interaction ( $P$

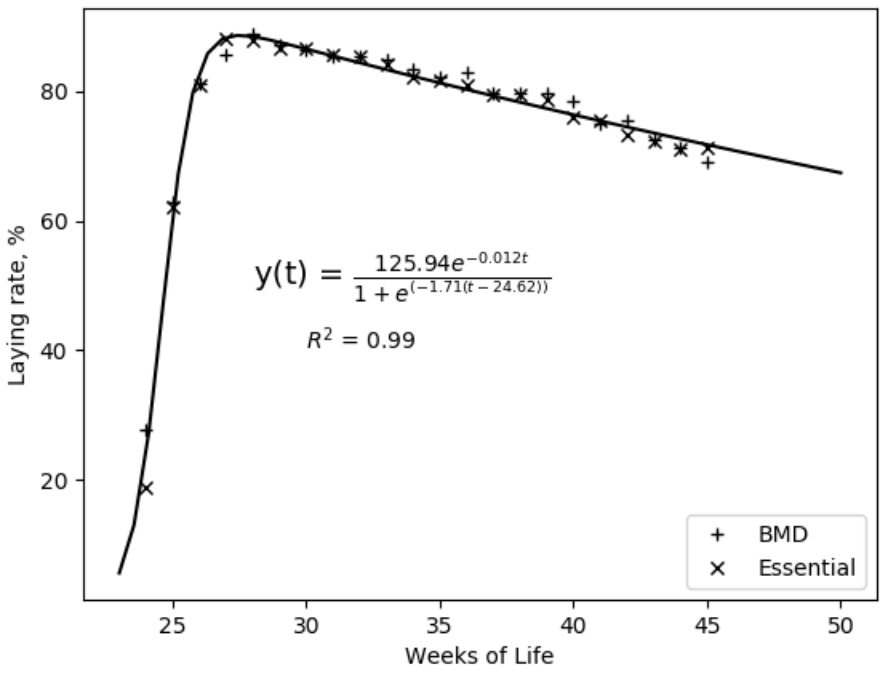

Figure 1 - Laying rates for breeders supplemented with either bacitracin methylene disalicylate (BMD) or functional oils (Essential, Oligo Basics Agroind. Ltda, Cascavel, Brazil) using a laying curve according to Yang et al., 1989.

$=0.07$ ), suggesting that fertility tended to increased by the functional oils at the beginning and the end of the experiment, but not during the peak production weeks (Figure 2). This may be related to a protective effect of the functional oils on the reproductive tract of the breeders by avoiding the lipid oxidation of the sperm (Breque et al., 2003). Although the roosters were not supplemented with functional oils, the antioxidant effect on the sperm may have taken place in the uterovaginal sperm-host glands. Hens are able to store sperm in the uterovaginal sperm-host and infundibular glands for a long period and can maintain the production of fertile eggs for several weeks (Bakst et al., 1994). However, the functional oils might have also had a direct and positive effect on the hens'

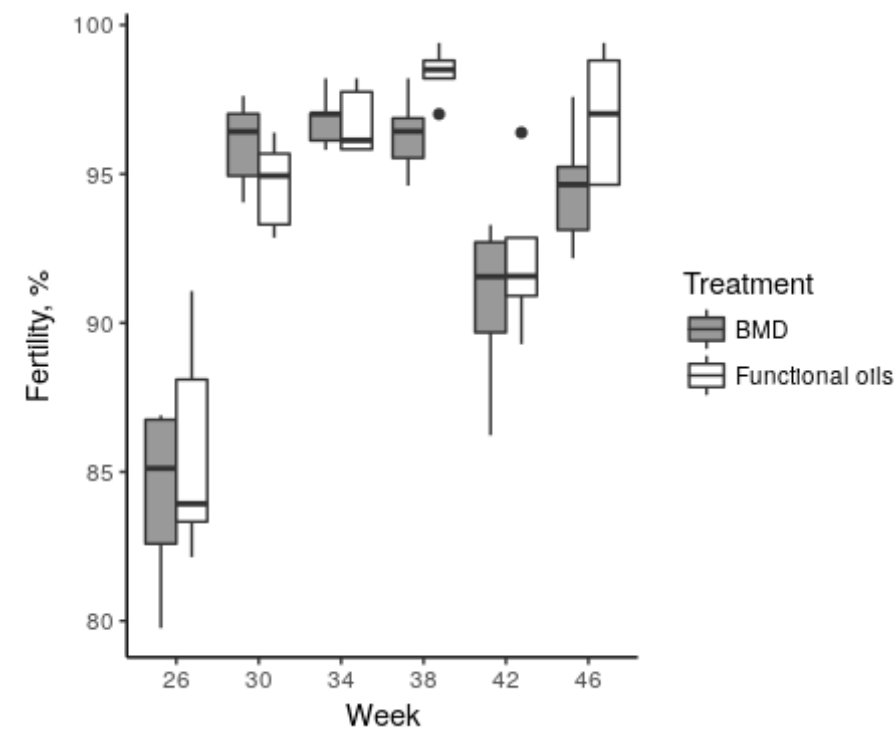

Figure $\mathbf{2}$ - Fertility (\%) of breeders supplemented with either bacitracin methylene disalicylate (BMD) or functional oils (Essential, Oligo Basics Agroind. Ltda, Cascavel, Brazil). 
Contini J, Schmidt J, Gonçalves D, Baldo J, Fernandes JIM, Torrent J
Suplementation of Antibiotic Growth Promoters versus Supplementation of Functional Oils in Broiler Breeders: Performance and Offspring Effects reproductive capabilities as the supplementation of functional oils also decreased embryonic mortality from $11.01 \%$ to $9.64 \% \quad(p<0.03)$ when compared to BMD. The chorioallantois, which is the respiratory organ that forms a network of capillaries in the internal membrane of the eggshell, is formed during this period (Moran, 2007) and the antioxidant capabilities of the embryo might have improved by the supplementation of the functional oils. Weekly breeders mortality was not affected by type of additive and averaged 0.13 $\pm 0.05 \%$ across treatments. The age of the breeders also affected the embryonic mortality $(p<0.01)$, which was higher at the beginning of the experiment (Figure 3). The percentage of chicks/breeder/day was $56.08 \%$ for the BMD and $57.62 \%$ for the functional oils supplemented breeders, but this difference was not significant $(p=0.63)$. As expected, chick weight increased with the age of the breeders $(p<0.01)$ but was not affected by the type of additive and averaged $43.0 \mathrm{~g}$, across treatments. The type of additive did not affect the percentage of either of contaminated or cracked eggs, being the average for these two parameters, 0.5 and $0.4 \%$, respectively.

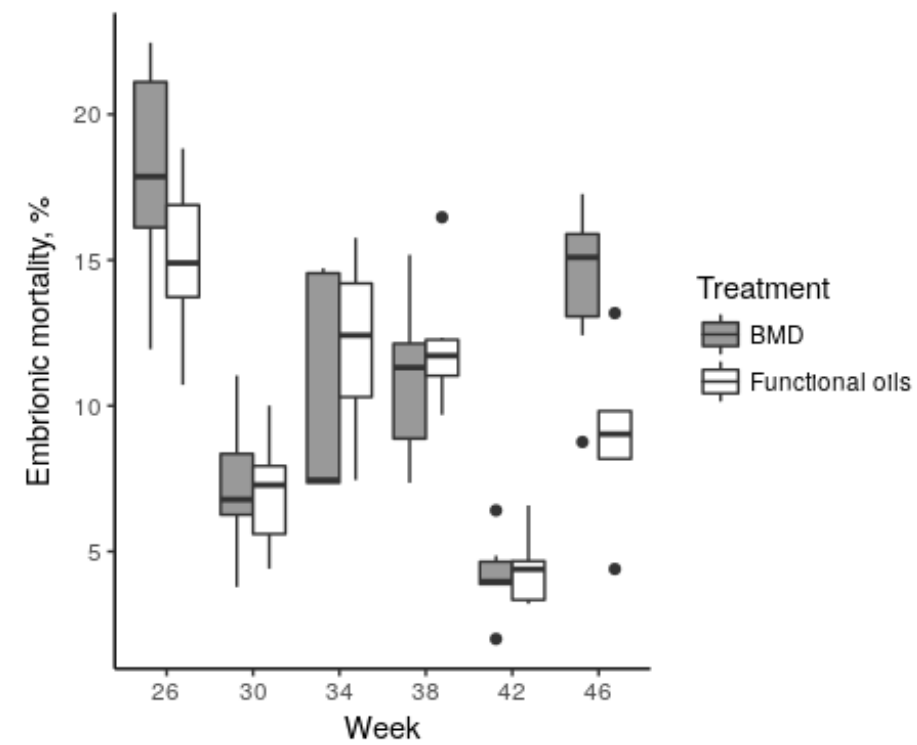

Figure 3 - Embryonic mortality (\%) of chicks from breeders supplemented with either bacitracin methylene disalicylate (BMD) or functional oils (Essential, Oligo Basics Agroind. Ltda, Cascavel, Brazil).

As expected, the age of the breeder increased all the studied egg characteristics $(p<0.01)$ other than shell thickness (Figure 4). However, the effects of the treatments on the egg characteristics were numerically minimal, being the averages across treatments, 61.3 $\mathrm{g}, 18.5 \mathrm{~g}, 38.0 \mathrm{~g}$, and $5.19 \mathrm{~g}$ for the weights of the egg, yolk, white, and shell, respectively, and $0.509 \mathrm{~mm}$ and 1.081 for the shell thickness and specific gravity, respectively.

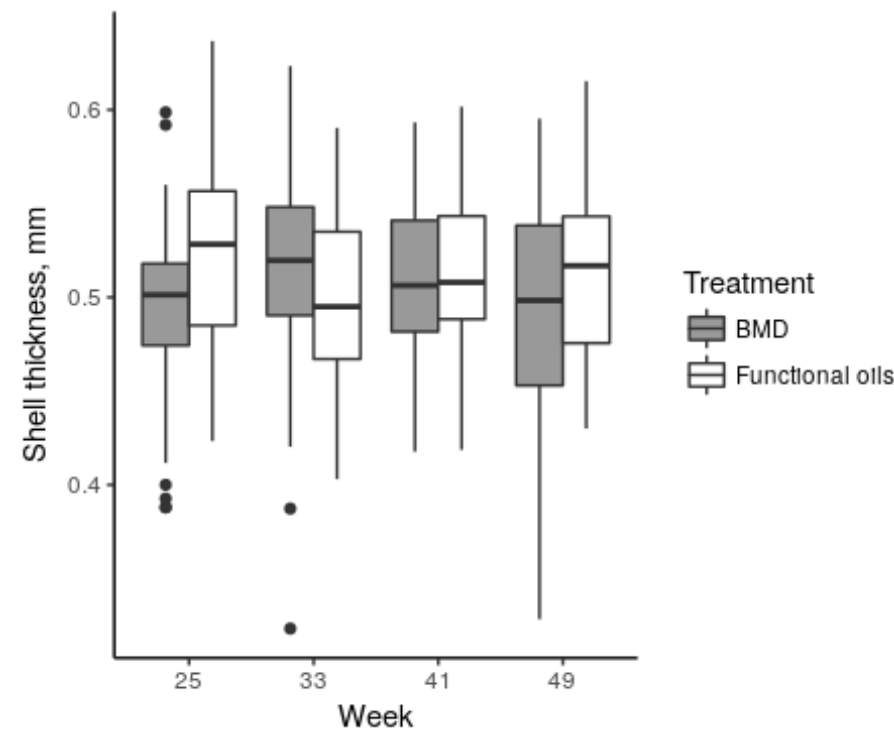

Figure 4 - Eggshell thickness (MM) for breeders supplemented with either bacitracin methylene disalicylate (BMD) or functional oils (Essential, Oligo Basics Agroind. Ltda, Cascavel, Brazil).

\section{Experiment II}

Performance results showed a maternal effect from 1 to 7 days of ages, where supplementation of breeders with functional oils did not result in different body weight gain $(0.128$ versus $0.132 \mathrm{~kg}$; $p=0.08$ ) but resulted in increased feed conversion rate (1.272 versus $1.227 \mathrm{~g}: \mathrm{g} ; p=0.02$ ). There was also an interaction between maternal and offspring effects $(p=0.01)$, where the supplementation of functional oils increased the feed conversion rate in the offspring supplemented with Enramycin but decreased it when the offspring were supplemented with functional oils (Table 3). However, the differences in performance disappeared between days 8 and 21, being the average across treatments $0.807 \mathrm{~kg}, 1.124 \mathrm{~kg}$, and $1.373 \mathrm{~g}: \mathrm{g}$ for body weight gain, feed intake, and feed conversion rate, respectively (Table 3 ). As there were no differences in feed intake, only differences in either digestibility or metabolizability could explain the higher feed conversion during the first week of the experiment for the offspring of functional oils supplemented breeders. Antibiotic growth promoters have been shown to delay the development of the gut microbiota when compared to probiotics (Gao et al., 2017), which may negatively affect performance. However, and unlike with antibiotic supplementation, functional oils supplementation has been shown to maintain the total number of bacteria in the intestinal microbiota (Moraes et al., 2019), so one could expect improved weight gains after the first week of age might be due to a better development of the gut microbiota. 
Table 3 - Offspring performance of bacitracin methylene disalicylate (BMD) or functional oils (FO: Essential, Oligo Basics Agroind. Ltda, Cascavel, Brazil) supplemented breeders, supplemented with either functional oils or Enramycin from 1 to $21 \mathrm{~d}$.

\begin{tabular}{|c|c|c|c|c|c|c|c|c|c|c|}
\hline \multirow{3}{*}{$\frac{\text { Item }}{1-7 \text { days }}$} & \multicolumn{8}{|c|}{ Offspring } & \multirow{2}{*}{$p$-value } & \multirow[b]{3}{*}{ Interactior } \\
\hline & \multirow[b]{2}{*}{ Maternal } & \multicolumn{2}{|c|}{ BMD $^{1}$} & \multicolumn{2}{|c|}{$\mathrm{FO}$} & \multicolumn{2}{|c|}{ Average } & \multirow[b]{2}{*}{ Maternal } & & \\
\hline & & Mean & SD & Mean & SD & Mean & SD & & Offspring & \\
\hline \multicolumn{11}{|c|}{ BW gain, kg } \\
\hline & Enramycin & 0.132 & 0.003 & 0.132 & 0.007 & 0.132 & 0.007 & & & \\
\hline & FO & 0.125 & 0.009 & 0.130 & 0.008 & 0.128 & 0.009 & & & \\
\hline & Average & 0.129 & 0.007 & 0.131 & 0.007 & 0.130 & 0.007 & 0.06 & 0.31 & 0.35 \\
\hline \multicolumn{11}{|c|}{ Feed Intake, kg } \\
\hline & Enramycin & 0.158 & 0.09 & 0.163 & 0.05 & 0.161 & 0.08 & & & \\
\hline & FO & 0.163 & 0.11 & 0.159 & 0.06 & 0.161 & 0.09 & & & \\
\hline & Average & 0.161 & 0.10 & 0.161 & 0.06 & 0.161 & 0.08 & 0.81 & 0.79 & 0.11 \\
\hline \multicolumn{11}{|l|}{ FCR, g:g } \\
\hline & Enramycin & 1.214 & 0.046 & 1.239 & 0.057 & 1.227 & 0.052 & & & \\
\hline & FO & 1.309 & 0.052 & 1.234 & 0.055 & 1.272 & 0.065 & & & \\
\hline & Average & 1.262 & 0.069 & 1.237 & 0.054 & 1.249 & 0.062 & 0.02 & 0.16 & 0.01 \\
\hline \multicolumn{11}{|c|}{$8-21$ days } \\
\hline \multicolumn{11}{|c|}{ BW gain, kg } \\
\hline & Enramycin & 0.807 & 0.034 & 0.797 & 0.030 & 0.802 & 0.022 & & & \\
\hline & FO & 0.818 & 0.026 & 0.804 & 0.024 & 0.811 & 0.030 & & & \\
\hline & Average & 0.813 & 0.027 & 0.801 & 0.027 & 0.807 & 0.026 & 0.59 & 0.18 & 0.81 \\
\hline \multicolumn{11}{|c|}{ Feed Intake, kg } \\
\hline & Enramycin & 1.130 & 0.050 & 1.106 & 0.046 & 1.118 & 0.048 & & & \\
\hline & FO & 1.133 & 0.058 & 1.124 & 0.061 & 1.129 & 0.058 & & & \\
\hline & Average & 1.132 & 0.052 & 1.115 & 0.053 & 1.124 & 0.053 & 0.85 & 0.36 & 0.67 \\
\hline \multicolumn{11}{|l|}{ FCR, g:g } \\
\hline & Enramycin & 1.372 & 0.035 & 1.379 & 0.031 & 1.375 & 0.032 & & & \\
\hline & FO & 1.353 & 0.075 & 1.390 & 0.036 & 1.371 & 0.060 & & & \\
\hline & Average & 1.363 & 0.058 & 1.384 & 0.033 & 1.373 & 0.048 & 0.80 & 0.19 & 0.35 \\
\hline
\end{tabular}

'Bacitracin methylene disalicylate.

Although the offspring of functional oils supplemented breeders gained $86 \mathrm{~g}$ more than the offspring of BMD supplemented breeders, the difference was not significant $(p=0.09)$. Feed intake, feed conversion rate, and mortality were $4.447 \mathrm{~kg}$, $1.565 \mathrm{~g}: \mathrm{g}$, and $3.75 \%$, across treatments (Table 4) and were not different. Also, and although one limitation of this research was the lack of a control treatment without the supplementation of any growth promoter, either antibiotic or not, our findings show that under the conditions of the trials, the functional oils can successfully substitute antibiotic growth promoters both in breeder and broiler diets. The positive effects seen on the breeders tended to carry over to their offspring, as birds were numerically heavier $(p=0.09)$ at 42 days of age. This carry-over, if confirmed by other researches, would demonstrate an epigenetic effect, which could be attributed to the antioxidant capacity of functional oils, as positive epigenetic effects have been reported for antioxidants in other species. For example, antioxidant supplementation in sows and rats resulted in enhanced offspring growth performance
(Wan et al., 2018) and improved offspring metabolic health (Zheng et al., 2019).

In conclusion, broiler breeder supplementation with functional oils was, at least, as effective as BMD supplementation. In our trial, functional oils decreased embryonic mortality during the first $72 \mathrm{~h}$ of incubation and did not negatively affect any other reproductive parameter. This positive effect seemed to be carried over to the offspring, as birds from breeders supplemented with functional oils were numerically heavier at $42 \mathrm{~d}$ of age. When the offspring were supplemented with functional oils, the effects on the performance were not different from those attained with Enramycin supplementation, showing that functional oils can successfully substitute antibiotic growth promoters both in broiler breeders and broilers.

\section{REFERENCES}

Avila VS de, Penz Jr AM, Rosa PS, Brum PAR de, Guidoni AL, Ledur MC Influence of feeding time on sexual maturity and carcass composition in female broiler breeders. Brazilian Journal of Poultry Science 2003;5(3):189-196. 
Table 4 - Offspring performance of bacitracin methylene disalicylate (BMD) or functional oils (FO: Essential, Oligo Basics Agroind. Ltda, Cascavel, Brazil) supplemented breeders, supplemented with either functional oils or Enramycin from 22 to $42 \mathrm{~d}$ and from 1 to $42 \mathrm{~d}$.

\begin{tabular}{|c|c|c|c|c|c|c|c|c|c|c|}
\hline \multirow{3}{*}{$\frac{\text { Item }}{22-42 \text { days }}$} & \multirow[b]{3}{*}{ Maternal } & \multicolumn{6}{|c|}{ Offspring } & \multirow{2}{*}{\multicolumn{3}{|c|}{$p$-value }} \\
\hline & & \multicolumn{2}{|c|}{$\mathrm{BMD}^{1}$} & \multicolumn{2}{|c|}{$\mathrm{FO}$} & \multicolumn{2}{|c|}{ Average } & & & \\
\hline & & Mean & SD & Mean & SD & Mean & SD & Maternal & Offspring & Interaction \\
\hline \multicolumn{11}{|l|}{ BW gain, kg } \\
\hline & Enramycin & 1.869 & 0.145 & 1.879 & 0.129 & 1.874 & 0.129 & & & \\
\hline & FO & 1.950 & 0.120 & 1.963 & 0.107 & 1.957 & 0.134 & & & \\
\hline & Average & 1.910 & 0.156 & 1.921 & 0.118 & 1.915 & 0.136 & 0.09 & 0.97 & 0.97 \\
\hline \multicolumn{11}{|c|}{ Feed Intake, kg } \\
\hline & Enramycin & 3.086 & 0.246 & 3.062 & 0.222 & 3.074 & 0.228 & & & \\
\hline & FO & 3.273 & 0.296 & 3.233 & 0.179 & 3.253 & 0.238 & & & \\
\hline & Average & 3.179 & 0.281 & 3.148 & 0.215 & 3.163 & 0.265 & 0.03 & 0.69 & 0.92 \\
\hline \multicolumn{11}{|l|}{ FCR, g:g } \\
\hline & Enramycin & 1.654 & 0.061 & 1.640 & 0.047 & 1.671 & 0.054 & & & \\
\hline & FO & 1.687 & 0.060 & 1.656 & 0.074 & 1.648 & 0.067 & & & \\
\hline & Average & 1.647 & 0.061 & 1.672 & 0.061 & 1.659 & 0.061 & 0.24 & 0.28 & 0.69 \\
\hline \multicolumn{11}{|l|}{$1-42$ days } \\
\hline \multicolumn{11}{|l|}{ BW gain, kg } \\
\hline & Enramycin & 2.808 & 0.152 & 2.808 & 0.140 & 2.808 & 0.142 & & & \\
\hline & FO & 2.893 & 0.181 & 2.897 & 0.114 & 2.895 & 0.147 & & & \\
\hline & Average & 2.851 & 0.168 & 2.853 & 0.132 & 2.852 & 0.149 & 0.09 & 0.97 & 0.97 \\
\hline \multicolumn{11}{|c|}{ Feed Intake, kg } \\
\hline & Enramycin & 4.374 & 0.297 & 4.330 & 0.264 & 4.352 & 0.274 & & & \\
\hline & FO & 4.569 & 0.353 & 4.516 & 0.235 & 4.542 & 0.292 & & & \\
\hline & Average & 4.471 & 0.332 & 4.423 & 0.261 & 4.447 & 0.295 & 0.06 & 0.62 & 0.96 \\
\hline \multicolumn{11}{|l|}{ FCR, g:g } \\
\hline & Enramycin & 1.560 & 0.044 & 1.584 & 0.026 & 1.572 & 0.036 & & & \\
\hline & FO & 1.549 & 0.053 & 1.568 & 0.051 & 1.559 & 0.051 & & & \\
\hline & Average & 1.555 & 0.049 & 1.576 & 0.040 & 1.565 & 0.045 & 0.15 & 0.37 & 0.87 \\
\hline \multicolumn{11}{|l|}{ Mortality, \% } \\
\hline & Enramycin & 4.20 & 0.04 & 3.30 & 0.02 & 3.75 & 0.03 & & & \\
\hline & FO & 3.30 & 0.03 & 4.20 & 0.04 & 3.75 & 0.04 & & & \\
\hline & Average & 3.75 & 0.04 & 3.75 & 0.03 & 3.75 & 0.03 & 0.66 & 0.95 & 0.79 \\
\hline
\end{tabular}

'Bacitracin methylene disalicylate.

Bakst MR, Wishart G, Brillard, JP. Oviducal sperm selection, transport and storage in poultry. Poultry Science Reviews 1994;5(5):117-143.

Bess F, Favero A, Vieira SL, Torrent J. The effects of functional oils on broiler diets of varying energy levels. Journal of Applied Poultry Research 2012;21:567-78

Birendra M, Jha R. Oxidative stress in the poultry gut: potential challenges and interventions. Frontiers in Veterinary Science 2019;6:60.

Bozkurt M, Alçiçek A, Çabuk M, Küçükyilmaz K, Çatli AU. Effect of an herbal essential oil mixture on growth, laying traits, and egg hatching characteristics of broiler breeders. Poultry Science 2009;88(11):23682374.

Breque C, Surai, PF, Brillard, JP. Roles of antioxidants on prolonged storage of avian spermatozoa in vivo and in vitro. Molecular Reproduction and Development 2003;66(3):314- 323.

Efron B, Tibshirani R. An introduction to the bootstrap. Boca Raton: Chapman \& Hall; 1993.

Gao P, Ma C, Sun Z, Wang L, Huang S, Su X, et al. Feed-additive probiotics accelerate yet antibiotics delay intestinal microbiota maturation in broiler chicken. Microbiome 2017;5:91.
Hu R, He Y, Arowolo MA, Wu S, He J. Polyphenols as potential attenuators of heat stress in poultry production. Antioxidants 2019;8(3):67.

Koedijk RM, Linde IB van de, Lamot DM, Hilbert M, Enting H. Antioxidants in broiler breeder diets can affect offspring performance. Proceedings of the 26 $6^{\text {th }}$ Australian Poultry Science Symposium; 2015 Feb 8-11; Sydney, Austrália. New South Wales: Poultry Research Foundation; 2015. p.194-197.

Lewis PD, Gous, RM. Constant and changing photoperiods in the laying period for broiler breeders allows normal or accelerated growth during the rearing period. Poultry Science 2006;85:321-325.

Moraes PO, Andretta I, Cardinal KM, Ceron M, Vilella L, Borille R, et al. Effect of functional oils on the immune response of broilers challenged with Eimeria spp. Animal 2019;13(10):2190-2198.

Moran Jr. ET. Nutrition of the developing embryo and hatchling. Poultry Science 2007;86(5):1043-1049.

Murakami AE, Eyng C, Torrent J. Effects of functional oils on coccidiosis and apparent metabolizable energy in broiler chickens. Asian-Australasian Journal of Animal Science 2014;27(7):981-989.

Novak AF, Clark GC, Dupuy HP. Antimicrobial activity of some ricinoleic and oleic acid derivatives. Journal of the American Oil Chemists' Society 1961;38:321-324. 
Pappas AC, Acamovic T, Sparks NHC, Surai PF, McDevitt RM. Effects of supplementing broiler breeder diets with organic selenium and polyunsaturated fatty acids on egg quality during storage, Poultry Science 2005;84(6):865-874.

R: The R Project for Statistical Computing [cited 2018 Oct 12]. Available from: www.r-project.org/.

Rosa AP, Scher A, Sorbara JOB, Boemo LS, Forgiarini J, Londero A. Effects of canthaxanthin on the productive and reproductive performance of broiler breeders. Poultry Science 2012;91(3):660-666.

Surai PF, Kochish II, Romanov MN, Griffin DK. Nutritional modulation of the antioxidant capacities in poultry:the case of vitamin E. Poultry Science 2019;98(9):4030-4041.

Torrent J, Arce Menocal J, López Coello C, Ávila González E. Effects of functional oils on performance and carcass characteristics of broilers under two ambient temperatures. Poultry Science 2019;98(11):58555861.
Trevisan MTS, Pfundstein B, Haubner R, Würtele G, Spiegelhalder B, Bartsch $\mathrm{H}$, et al. Characterization of alkyl phenols in cashew (Anacardium occidentale) products and assay of their antioxidant capacity. Food and Chemical Toxicology 2005;44(2):188-197.

Vieira C, Fetzer S, Sauer SK, Evangelista S, Averbeck B, Kress M, et al. Pro- and anti-inflammatory actions of ricinoleic acid:similarities and differences with capsaicin. Naunyn-Schmiedeberg's Archives of Pharmacology 2001;364:87-95.

, McMillan, I.Wan J, Xu Q, He J. Maternal chitosan oligosaccharide supplementation during late gestation and lactation affects offspring growth. Italian Journal of Animal Science 2018;17:4:994-1000.

Yang N, Wu C, McMillan, I. New mathematical model of poultry egg production. Poultry Science 1989;68(4):476-481.

Zheng S, Feng Q, Cheng J, Zheng J. Maternal resveratrol consumption and its programming effects on metabolic health in offspring mechanisms and potential implications. Bioscience Reports 2018;38(2):BSR20171741. 
